\title{
Genomic Investigations of Evolutionary Dynamics and Epistasis in Microbial Evolution Experiments
}

\author{
Elizabeth R. Jerison ${ }^{1}$ and Michael M. Desai ${ }^{*}$ \\ ${ }^{1}$ Department of Organismic and Evolutionary Biology, Department of Physics, and FAS Center for Systems Biology, Harvard \\ University, Cambridge MA 02138
}

\begin{abstract}
Microbial evolution experiments enable us to watch adaptation in real time, and to quantify the repeatability and predictability of evolution by comparing identical replicate populations. Further, we can resurrect ancestral types to examine changes over evolutionary time. Until recently, experimental evolution has been limited to measuring phenotypic changes, or to tracking a few genetic markers over time. However, recent advances in sequencing technology now make it possible to extensively sequence clones or whole-population samples from microbial evolution experiments. Here, we review recent work exploiting these techniques to understand the genomic basis of evolutionary change in experimental systems. We first focus on studies that analyze the dynamics of genome evolution in microbial systems. We then survey work that uses observations of sequence evolution to infer aspects of the underlying fitness landscape, concentrating on the epistatic interactions between mutations and the constraints these interactions impose on adaptation.
\end{abstract}

*To whom correspondence may be addressed: mmdesai@fas.harvard.edu 


\section{Introduction}

Laboratory evolution experiments complement studies of natural populations, by making it possible to more directly investigate the underlying factors that shape observed patterns of genetic diversity. While these experiments neglect many important complexities of the natural world, they offer a number of advantages. In experimental systems, we can observe evolutionary dynamics in real time, and exploit a "frozen fossil record" to resurrect ancestral types and directly compare them to their descendants. We can also maintain many populations in parallel, and replay the tape of life thousands of times in identical (or different) conditions. Finally, we can tune key evolutionary parameters such as population sizes and mutation rates, and assess their importance with other parameters held constant.

A large body of work has exploited these advantages to investigate how evolutionary history, chance, and natural selection influence evolutionary outcomes [1,2]. For example, recent work has examined how the distribution of fitness effects of available mutations determines the power of natural selection [3,4]. Additionally, multiple studies have analyzed the role of epistasis in creating 'historical contingency,' where an initial mutation constrains or potentiates future evolution [5-7]. Finally, laboratory evolution has been used to investigate how parameters such as population size affect evolutionary dynamics $[8,9]$. All of these studies aim to describe how features of the evolutionary landscape combine to determine evolutionary outcomes - a general process that is not confined to laboratory systems. When used for this purpose, laboratory microbial evolution experiments have the potential to be a model system for understanding the structure and diversity of genomes.

Until recently, experimental evolution has been limited primarily to phenotypic measurements. Numerous studies have examined how fitness changes over time, and how the rate of adaptation depends on factors such as the population size, initial genotype, population structure, or environmental conditions. Many studies have also tracked the frequencies of observable markers (e.g. drug resistance or fluorescent reporters) through time $[3,4,10,11]$ to draw inferences $[3,12,13]$ about the evolutionary process. In recent years, however, advances in sequencing technology have made it possible to sequence clones or whole-population samples from hundreds of parallel experimental lines [14-16]. In this review, we summarize recent work that has begun to apply these technical advances to long-term laboratory evolution experiments, to directly observe how microbial genomes evolve in the laboratory. We focus particularly on the influence of epistasis on genome evolution (for a more general and comprehensive review, see [17]).

\section{The dynamics of adaptation}

The population genetic forces of mutation, selection, and drift govern the dynamics of genome evolution, determining which mutations will survive competition to fix in the population, and the signatures this process will leave on patterns of genetic diversity. An extensive population genetics literature has explored these connections theoretically. However, until recently it has been impossible to directly observe these dynamics in laboratory populations. Empirical studies of this type are necessary to determine which theoretically proposed regimes evolving populations actually experience. For example, is adaptation typically mutation limited, and hence in a regime where beneficial mutations fix independently, on their own merits? Or do beneficial mutations typically arise in multiple different lineages, resulting in a "clonal interference" regime where many mutations compete for dominance? Studies that directly observe genome evolution in real time can distinguish between these and other possibilities. They can also point the way to complications that theory has overlooked, but that may be essential to the evolutionary process.

The first experiments to directly observe genomic evolution were in bacteriophage, where several studies Sangersequenced individual phage clones at multiple timepoints in several replicate populations to describe patterns of parallel and convergent evolution [18-23]. Next-generation sequencing now makes similar studies possible in microbial populations. Recent studies have exploited both whole-population ("metagenomic") sequencing of samples isolated at multiple timepoints from one or more evolving lines, and also sequencing of clones, to understand genomic evolution in 
these populations. These approaches offer somewhat different perspectives on the dynamics of molecular evolution (Figure 1).

The first major study to examine the dynamics of genome evolution in microbial laboratory populations focused on a single line of a long-term evolution experiment in E. coli. By analyzing a clone sampled from each of 5 timepoints during 20,000 generations of adaptation, Barrick and Lenski [24] showed that mutations continue to accumulate steadily through time despite a dramatic slowdown in the rate at which fitness increases. This divergence between phenotypic and sequence-level evolution points to the potential importance of epistasis in shaping adaptation [25-27]. More recent studies have sequenced clones isolated from multiple timepoints in several replicate populations [28,29]. This work demonstrates that adaptation is typically not mutation-limited: instead, there is clonal interference between competing beneficial mutations. Clonal interference affects which mutations fix in the population, and hence influences both molecular diversity and the dynamics of adaptation. In particular, the competition among beneficial mutations ensures that many are wasted, reducing the efficiency of selection and the predictability of molecular evolution. Clonal interference also affects the efficacy of other evolutionary processes, such as indirect selection on mutation rates [30].

Sequencing whole-population samples through time in multiple replicate populations offers an alternative view of the dynamics of adaptation [31-33]. For example, one large-scale study in budding yeast highlighted the importance of pervasive clonal interference and hitchhiking in determining the outcomes of adaptation and in limiting the degree of parallelism in the genome evolution of replicate lines [31]. Other studies have shown that frequency-dependent interactions can evolve spontaneously and play an important role in both lineage dynamics and the maintenance of genomic diversity [34,35]. Finally, while most laboratory evolution experiments start from clonal populations, and hence focus entirely on evolution from new mutations, another body of work has used whole-population timecourse sequencing to analyze the dynamics of adaptation from standing genetic variation. Much of this work is carried out in Drosophila [3638], but recent work has addressed similar questions using extensive sequencing of yeast populations founded from outcrossed lines [39].

\section{Epistasis, historical contingency, and the structure of the landscape}

Genome evolution is shaped both by the dynamics of the evolutionary process and by the spectrum of available mutations and their fitness effects (i.e. the "fitness landscape"). Thus we can use observations of genome evolution as a tool to infer relevant aspects of the landscape. Here we focus on recent progress in characterizing patterns of epistasis (i.e. how the fitness effects of mutations depend on the genetic background), and in understanding how epistasis constrains evolution. In principle, acquiring one mutation can change the entire distribution of fitness effects of future mutations. This "macroscopic epistasis" summarizes many underlying details of individual genetic interactions [26], and can lead to historical contingency where an initial mutation constrains the future course of adaptation. Even without extensive macroscopic epistasis, interactions among specific sets of mutations ("microscopic epistasis") can affect the mutational trajectories open to an evolving population, and hence lead to historical contingency in patterns of molecular evolution. Microbial experimental evolution is particularly well-suited to probing patterns of both macroscopic and microscopic epistasis, because replicate populations will probabilistically explore available areas of the local landscape, and mutations that arise during evolution can be reconstructed in arbitrary combinations. Extensive replication also makes it possible to conduct large-scale studies of the mutations that occur during evolution and their statistical associations with one another, providing another type of evidence for epistasis [40].

Recent work has explored whether patterns of epistasis reflect the modularity of cellular organization. Within the smallest modular unit, a single gene, studies of the antibiotic resistance enzyme beta lactamase [41] and comprehensive reconstructions of mutations within a 9 amino acid region in yeast Hsp90 [42,43] have revealed widespread epistasis. This includes sign epistasis, in which a mutation shifts from beneficial to deleterious (or vice versa) depending on the genetic background it occurs in. Epistatic interactions may also occur at the protein complex or pathway level. In a landmark paper, Tenaillon et. al. [44] demonstrated such an effect in E. coli adapting to heat stress. By evolving 114 replicate 
populations and sequencing their genomes after evolution, this study uncovered two mutually exclusive routes to heat adaptation: populations acquired mutations either in the RNA polymerase pathway or in the termination factor rho, but not both [44,45] (see also [28] for a related effect). Further, in a single line there were often multiple mutations in one or the other pathway. To explore epistasis at the pathway level more broadly, two recent studies in $E$. coli and yeast evolved replicate populations with particular gene deletions, and found an enrichment in compensatory mutations targeting complexes associated with the deleted gene [46,47]. Together these studies indicate that a mutation in a particular pathway, either beneficial or deleterious, may potentiate beneficial mutations in this pathway. In contrast, within a single gene, one mutation is more likely to exclude further changes.

Several recent studies have found indications of a different, broader form of epistasis. In viruses, bacteria, and yeast, fitter genotypes reliably adapt more slowly than less-fit genotypes [5,27,48-51]. This slowdown indicates that the distribution of fitness effects of available beneficial mutations depends on genotype, either because there are fewer beneficial mutations available to fitter genotypes, or because the beneficial mutations have weaker effects ("diminishing returns epistasis"). Studies in E. coli and methylobacterium reconstructed all combinations of 4 or 5 mutations from an adaptive walk, finding a general pattern of diminishing returns [52,53]. More recently, Kryazhimskiy et. al. [5] used 640 replicate yeast populations to show that the rate of adaptation declines systematically with the fitness of the initial genotype - not because more-fit populations run out of beneficial mutations, but rather because the effects of beneficial mutations become weaker in fitter backgrounds. This effect is largely independent of the specific mutations involved, indicating a potentially general negative coupling among beneficial mutations.

Occasionally more dramatic effects of epistasis arise, where a mutation potentiates a major shift in the DFE, sometimes including changes to the population structure. The most extensively studied example of such an effect is from Richard Lenski's long-term evolution experiment in E. coli. In one of 12 replicate lines, after about 30,000 generations one lineage evolved the ability to utilize citrate under aerobic conditions [6]. This lineage stably coexisted for thousands of generations with the wild-type Cit- lineage, indicating the presence of frequency-dependent selection. Recent work has described the genetic basis of this transition, including multiple potentiating mutations [54,55]. While this example is so far unique, the effects of epistasis in potentiating the evolution of coexisting lineages have also been observed in another replicate population from the same experiment [56]. In both of these cases, historical contingency was essential: multiple interacting mutations are required to create the new phenotype. Related examples where sign epistasis limits potential evolutionary trajectories, leading to important effects of historical contingency, have been described in other systems $[57,58]$.

Larger-scale genomic changes, including shifts in ploidy, amplifications, deletions, inversions, copy number variation, and transposon-mediated insertions, can also represent an important aspect of the fitness landscape. Due to the limitations of short-read sequencing technology, these events have been comparatively overlooked in many laboratory evolution experiments [59]. However, they are a potential avenue for rapid innovation [59-62], and can clearly have dramatic effects on the future course of adaptation (e.g. they can lead to substantial macroscopic epistasis). The rates at which these events occur and the selective forces that act on them remain incompletely understood. For example, ploidy is clearly tuned rapidly by natural selection [63]. However, while recent studies find that experimental budding yeast populations quickly converge towards diploidy, these changes do not always have clear selective advantages [64-68]. In addition to changes in ploidy, amplifications or deletions of individual chromosomes (or parts of chromosomes) can also be rapidly selected, particularly in stressful conditions [69-74] or when starting from novel genotypes [75,76]. These amplifications or deletions can change the local fitness landscape, opening new avenues for adaptation. For example, a study of yeast adapting to high temperature [69] demonstrated that the initial phases of adaptation often involve a duplication of chromosome III. This duplication eventually reverts, and is replaced by a more "refined” solution that upregulates only specific genes on this chromosome. Copy number variation can also provide a rapid avenue to dosage adjustment. For example, studies of yeast in a variety of nutrient-limited conditions have found adaptive amplifications of transporters of the limiting nutrient [32,73,74]. 


\section{Conclusions and Future Directions}

Experimental evolution makes it possible to observe genome evolution in real time in a controlled, replicated laboratory setting. It also allows us to directly measure (and in some cases control) the underlying evolutionary parameters that influence which mutations fix and hence how genomes evolve. With the advent of inexpensive high-throughput sequencing, it has become possible to examine the dynamics of new mutations within adapting populations in unprecedented detail. Although experimental systems cannot reproduce the complexities of natural populations, the same rules of population genetics govern evolutionary dynamics in both systems, and so the dynamics observed in laboratory populations may be common to more complex natural cases. For example, recent studies have demonstrated the prevalence of clonal interference in microbial laboratory populations; this regime may be common in natural systems, such as Pseudomonas infections in the lungs of cystic fibrosis patients [77]. Recently, studies have also begun moving beyond the typical isolated population of laboratory evolution, to examine the emergence and maintenance of population structure [34,35,78-85]. In these studies, frequency-dependent selection gives rise to stable ecotypes readily even in very simple laboratory environments. Understanding the prevalence, dynamics, and long-term fate of these structured populations will help bridge the gap between the rich ecology of the natural world and the simplicity of laboratory evolution.

Recent progress has also been made towards understanding the structure of the fitness landscape, which plays a key role in determining evolutionary dynamics and patterns of genomic diversity. In particular, recent work has shown that diminishing-returns epistasis (where beneficial mutations generally damp each others' effects) may be a general feature of adaptation. Studies have also begun to examine how cellular organization influences the mutations that fix during evolution. Future work will likely bring increasing power to understand the fitness landscape and the cell via statistical associations between mutations that fix during evolution. New approaches, such as massive barcoding systems [86], also promise to provide a yet more detailed view of evolutionary dynamics, and also to significantly increase power to understand epistasis. These systems offer the prospect of measuring the dependence of the distribution of fitness effects on both genotype and environment, as well as the interactions between individual mutations.

To date, most microbial laboratory experiments have used model organisms, notably E. coli and S. cerevisiae. While the tractable genetics in these systems provides a strong advantage, in the future it will also be important to investigate the evolutionary process in a wider variety of organisms. This breadth will be necessary to help us distinguish which features of the evolutionary process are general, rather than specific to common laboratory models. Early progress has been made in describing mutational processes [87-89] and evolutionary outcomes [35,76,90] across a broader variety of microbes. Further studies along these lines will provide a more comprehensive view, and help connect with clinical and industrial applications where specific non-model microbes may be of particular interest.

Finally, the last few years has seen an explosion of data on microbial communities in nature and their roles in animal and plant health. However, relatively little is known about evolution in these complex environments. Studying evolution in these scenarios, and the interplay between the host and the community, is an exciting avenue of future research (e.g. see [29] for a pioneering study).

\section{Acknowledgements}

This work was supported by an NSF graduate research fellowship (ERJ), and by the James S. McDonnell Foundation, the Alfred P. Sloan Foundation, NSF grant PHY 1313638, and NIH grant GM104239 (MMD). 


\section{Figure Caption}

Figure 1: Schematic of the evolutionary dynamics in a microbial evolution experiment. (A) Muller diagram depicting the frequencies of each genotype in the population over time. Only lineages that reach substantial frequency are shown (many lower-frequency lineages will typically also exist). (B) Allele frequencies in the population from (A), as they would be measured using whole-population metagenomic sequencing. This strategy reveals the dynamics of major alleles, but lowfrequency mutations are undetectable. This metagenomic data also yields incomplete haplotype information: it is not always clear which mutations arise on which genetic backgrounds. (C) A phylogenetic tree built from clones that could be sampled from the population in (A; black dots). Colored boxes show the major mutations pictured in (A) and (B); grey boxes show 'private' mutations shared only by this clone and close relatives. This clone sequencing approach can be used to measure mutation rates and genetic diversity statistics such as heterozygosity, but provides limited information about allele frequencies over time. 


\section{References Cited}

1. Elena SF, Lenski RE: Evolution experiments with microorganisms: the dynamics and genetic bases of adaptation. Nat Rev Genet 2003, 4:457-469.

2. Buckling A, Craig Maclean R, Brockhurst MA, Colegrave N: The Beagle in a bottle. Nature 2009, 457:824-829.

3. Frenkel EM, Good BH, Desai MM: The Fates of Mutant Lineages and the Distribution of Fitness Effects of

Beneficial Mutations in Laboratory Budding Yeast Populations. Genetics 2014, 196:1217-1226.

4. Hegreness M, Shoresh N, Hartl D, Kishony R: An equivalence principle for the incorporation of favorable mutations in asexual populations. Science 2006, 311:1615-1617.

5. Kryazhimskiy S, Rice DP, Jerison ER, Desai MM: Global epistasis makes adaptation predictable despite sequencelevel stochasticity. Science 2014, 344:1519-1522. *This study shows that a global pattern of diminishing returns epistasis leads to convergent fitness evolution in laboratory budding yeast populations, despite diversification at the genome level. .

6. Blount ZD, Borland CZ, Lenski RE: Historical contingency and the evolution of a key innovation in an experimental population of Escherichia coli. Proc Natl Acad Sci U S A 2008, 105:7899-7906.

7. Travisano M, Mongold J, Bennett A, Lenski R: Experimental tests of the roles of adaptation, chance, and history in evolution. Science 1995, 267:87-90.

8. de Visser JAGM, Zeyl CW, Gerrish PJ, Blanchard JL, Lenski RE: Diminishing Returns from Mutation Supply Rate in Asexual Populations. Science 1999, 283:404-406.

9. Desai MM, Fisher DS, Murray AW: The Speed of Evolution and Maintenance of Variation in Asexual Populations. Current Biology 2007, 17:385-394.

10. Kao KC, Sherlock G: Molecular characterization of clonal interference during adaptive evolution in asexual populations of Saccharomyces cerevisiae. Nat Genet 2008, 40:1499-1504.

11. Lang GI, Botstein D, Desai MM: Genetic variation and the fate of beneficial mutations in asexual populations. Genetics 2011, 188:647-661.

12. Zhang W, Sehgal V, Dinh DM, Azevedo RBR, Cooper TF, Azencott R: Estimation of the rate and effect of new beneficial mutations in asexual populations. Theoretical Population Biology 2012, 81:168-178.

13. Moura de Sousa JA, Campos PRA, Gordo I: An ABC Method for Estimating the Rate and Distribution of Effects of Beneficial Mutations. Genome Biology and Evolution 2013, 5:794-806.

14. Dettman JR, Rodrigue N, Melnyk AH, Wong A, Bailey SF, Kassen R: Evolutionary insight from whole-genome sequencing of experimentally evolved microbes. Molecular Ecology 2012, 21:2058-2077.

15. Brockhurst MA, Colegrave N, Rozen DE: Next-generation sequencing as a tool to study microbial evolution. Molecular Ecology 2011, 20:972-980.

16. Lang GI, Desai MM: The spectrum of adaptive mutations in experimental evolution. Genomics 2014, 104:412416.

17. Barrick JE, Lenski RE: Genome dynamics during experimental evolution. Nature reviews. Genetics 2013, 14:827839.

18. Bollback JP, Huelsenbeck JP: Clonal interference is alleviated by high mutation rates in large populations. Mol Biol Evol 2007, 24:1397-1406.

19. Bollback JP, Huelsenbeck JP: Parallel genetic evolution within and between bacteriophage species of varying degrees of divergence. Genetics 2009, 181:225-234.

20. Betancourt AJ: Genomewide Patterns of Substitution in Adaptively Evolving Populations of the RNA Bacteriophage MS2. Genetics 2009, 181:1535-1544.

21. Bull JJ, Badgett MR, Wichman HA, Huelsenbeck JP, Hillis DM, Gulati A, Ho C, Molineux IJ: Exceptional convergent evolution in a virus. Genetics 1997, 147:1497-1507.

22. Wichman HA, Badgett MR, Scott LA, Boulianne CM, Bull JJ: Different trajectories of parallel evolution during viral adaptation. Science 1999, 285:422-424.

23. Miller CR, Joyce P, Wichman HA: Mutational Effects and Population Dynamics During Viral Adaptation Challenge Current Models. Genetics 2011, 187:185-202.

24. Barrick JE, Yu DS, Yoon SH, Jeong H, Oh TK, Schneider D, Lenski RE, Kim JF: Genome evolution and adaptation in a long-term experiment with Escherichia coli. Nature 2009, 461:1243-1247. **An analysis of mutations identified in clones from five timepoints spanning 20000 generation of laboratory adaptation in E. coli, and one additional clone from generation 40000, after the fixation of a mutator allele. .

25. Barrick JE, Lenski RE: Genome-wide mutational diversity in an evolving population of Escherichia coli. Cold Spring Harb Symp Quant Biol 2009, 74:119-129. 
26. Good BH, Desai MM: The Impact of Macroscopic Epistasis on Long-Term Evolutionary Dynamics. Genetics 2015, 199:177-190.

27. Wiser MJ, Ribeck N, Lenski RE: Long-Term Dynamics of Adaptation in Asexual Populations. Science 2013, 342:1364-1367.

28. Kvitek DJ, Sherlock G: Reciprocal sign epistasis between frequently experimentally evolved adaptive mutations causes a rugged fitness landscape. PLoS Genet 2011, 7:e1002056.

29. Barroso-Batista J, Sousa A, Lourenço M, Bergman M-L, Sobral D, Demengeot J, Xavier KB, Gordo I: The First Steps of Adaptation of <italic >Escherichia coli</italic> to the Gut Are Dominated by Soft Sweeps. PLoS Genet 2014, 10:e1004182.

30. Wielgoss S, Barrick JE, Tenaillon O, Wiser MJ, Dittmar WJ, Cruveiller S, Chane-Woon-Ming B, Medigue C, Lenski $\mathrm{RE}$, Schneider $\mathrm{D}$ : Mutation rate dynamics in a bacterial population reflect tension between adaptation and genetic load. Proc Natl Acad Sci U S A 2013, 110:222-227.

31. Lang GI, Rice DP, Hickman MJ, Sodergren E, Weinstock GM, Botstein D, Desai MM: Pervasive genetic hitchhiking and clonal interference in forty evolving yeast populations. Nature 2013, 500:571-574. **This study uses whole-population (metagenomic) sequencing to track the frequency through time of spontaneously arising mutations in forty replicate budding yeast populations. .

32. Kvitek DJ, Sherlock G: Whole genome, whole population sequencing reveals that loss of signaling networks is the major adaptive strategy in a constant environment. PLoS Genet 2013, 9:e1003972.

33. Maddamsetti R, Lenski RE, Barrick JE: Adaptation, Clonal Interference, and Frequency-Dependent Interactions in a Long-Term Evolution Experiment with Escherichia coli. Genetics 2015.

34. Herron MD, Doebeli M: Parallel Evolutionary Dynamics of Adaptive Diversification in <italic $>$ Escherichia coli</italic>. PLoS Biol 2013, 11:e1001490.

35. Traverse CC, Mayo-Smith LM, Poltak SR, Cooper VS: Tangled bank of experimentally evolved Burkholderia biofilms reflects selection during chronic infections. Proc Natl Acad Sci U S A 2013, 110:E250-259. *Detailed analysis of the evolutionary dynamics of ecological diversification in experimental Burkholderia biofilms. .

36. Burke MK, Dunham JP, Shahrestani P, Thornton KR, Rose MR, Long AD: Genome-wide analysis of a long-term evolution experiment with Drosophila. Nature 2010, 467:587-590.

37. Turner TL, Stewart AD, Fields AT, Rice WR, Tarone AM: Population-Based Resequencing of Experimentally Evolved Populations Reveals the Genetic Basis of Body Size Variation in <italic $>$ Drosophila melanogaster</italic>. PLoS Genet 2011, 7:e1001336.

38. Zhou D, Udpa N, Gersten M, Visk DW, Bashir A, Xue J, Frazer KA, Posakony JW, Subramaniam S, Bafna V, et al.: Experimental selection of hypoxia-tolerant Drosophila melanogaster. Proceedings of the National Academy of Sciences 2011, 108:2349-2354.

39. Burke MK, Liti G, Long AD: Standing genetic variation drives repeatable experimental evolution in outcrossing populations of Saccharomyces cerevisiae. Mol Biol Evol 2014, 31:3228-3239. *Whole-genome sequencing to analyze adaptation from standing variation in experimental budding yeast populations. .

40. Desai MM: Statistical questions in experimental evolution. Journal of Statistical Mechanics: Theory and Experiment 2013, 2013:P01003.

41. Weinreich DM, Delaney NF, Depristo MA, Hartl DL: Darwinian evolution can follow only very few mutational paths to fitter proteins. Science 2006, 312:111-114. **This study demonstrated the importance of sign epistasis in limiting the mutational trajectories by which beta lactamase can evolve to improve drug resistance. .

42. Hietpas RT, Jensen JD, Bolon DNA: Experimental illumination of a fitness landscape. Proceedings of the National Academy of Sciences 2011, 108:7896-7901.

43. Bank C, Hietpas RT, Jensen JD, Bolon DNA: A Systematic Survey of an Intragenic Epistatic Landscape. Mol Biol Evol 2015, 32:229-238.

44. Tenaillon O, Rodriguez-Verdugo A, Gaut RL, McDonald P, Bennett AF, Long AD, Gaut BS: The molecular diversity of adaptive convergence. Science 2012, 335:457-461. **An important study analyzing the variation in the molecular basis of adaptation in 114 replicate lines of E. coli adapted to high temperature for 2000 generations, highlighting an important role for epistasis. .

45. Rodríguez-Verdugo A, Carrillo-Cisneros D, González-González A, Gaut BS, Bennett AF: Different tradeoffs result from alternate genetic adaptations to a common environment. Proceedings of the National Academy of Sciences 2014, 111:12121-12126.

46. Blank D, Wolf L, Ackermann M, Silander OK: The predictability of molecular evolution during functional innovation. Proc Natl Acad Sci U S A 2014, 111:3044-3049.

47. Szamecz B, Boross G, Kalapis D, Kovács K, Fekete G, Farkas Z, Lázár V, Hrtyan M, Kemmeren P, Groot Koerkamp MJA, et al.: The Genomic Landscape of Compensatory Evolution. PLoS Biol 2014, 12:e1001935. 
48. Couce A, Tenaillon OA: The rule of declining adaptability in microbial evolution experiments. Frontiers in Genetics 2015, 6:99.

49. Silander OK, Tenaillon O, Chao L: Understanding the Evolutionary Fate of Finite Populations: The Dynamics of Mutational Effects. PLoS Biol 2007, 5:e94.

50. Perfeito L, Sousa A, Bataillon T, Gordo I: Rates of fitness decline and rebound suggest pervasive epistasis. Evolution 2014, 68:150-162.

51. Barrick JE, Kauth MR, Strelioff CC, Lenski RE: Escherichia coli rpoB mutants have increased evolvability in proportion to their fitness defects. Mol Biol Evol 2010, 27:1338-1347.

52. Chou HH, Chiu HC, Delaney NF, Segre D, Marx CJ: Diminishing returns epistasis among beneficial mutations decelerates adaptation. Science 2011, 332:1190-1192.

53. Khan AI, Dinh DM, Schneider D, Lenski RE, Cooper TF: Negative epistasis between beneficial mutations in an evolving bacterial population. Science 2011, 332:1193-1196.

54. Blount ZD, Barrick JE, Davidson CJ, Lenski RE: Genomic analysis of a key innovation in an experimental Escherichia coli population. Nature 2012, 489:513-518. **This study analyzes the molecular basis of the evolution of citrate utilization in an experimental E. coli population, demonstrating a key role for historical contingency. .

55. Quandt EM, Deatherage DE, Ellington AD, Georgiou G, Barrick JE: Recursive genomewide recombination and sequencing reveals a key refinement step in the evolution of a metabolic innovation in Escherichia coli. Proc Natl Acad Sci U S A 2014, 111:2217-2222.

56. Plucain J, Hindré T, Le Gac M, Tenaillon O, Cruveiller S, Médigue C, Leiby N, Harcombe WR, Marx CJ, Lenski RE, et al.: Epistasis and Allele Specificity in the Emergence of a Stable Polymorphism in Escherichia coli. Science 2014, 343:1366-1369.

57. Carroll SM, Lee M-C, Marx CJ: Sign epistasis limits evolutionary trade-offs at the confluence of single- and multi-carbon metabolism in Methylobacterium Extorquens AM1. Evolution 2014, 68:760-771.

58. de Vos MGJ, Poelwijk FJ, Battich N, Ndika JDT, Tans SJ: Environmental Dependence of Genetic Constraint. PLoS Genet 2013, 9:e1003580.

59. Deatherage DE, Traverse CC, Wolf LN, Barrick JE: Detecting rare structural variation in evolving microbial populations from new sequence junctions using breseq. Frontiers in Genetics 2015, 5.

60. Raeside C, Gaffé J, Deatherage DE, Tenaillon O, Briska AM, Ptashkin RN, Cruveiller S, Médigue C, Lenski RE, Barrick JE, et al.: Large Chromosomal Rearrangements during a Long-Term Evolution Experiment with Escherichia coli. mBio 2014, 5.

61. Renda BA, Dasgupta A, Leon D, Barrick JE: Genome Instability Mediates the Loss of Key Traits by Acinetobacter baylyi ADP1 during Laboratory Evolution. J Bacteriol 2015, 197:872-881.

62. Barrick JE, Colburn G, Deatherage DE, Traverse CC, Strand MD, Borges JJ, Knoester DB, Reba A, Meyer AG: Identifying structural variation in haploid microbial genomes from short-read resequencing data using breseq. BMC Genomics 2014, 15:1039.

63. Paquin C, Adams J: Frequency of fixation of adaptive mutations is higher in evolving diploid than haploid yeast populations. Nature 1983, 302:495-500.

64. Gerstein AC, McBride RM, Otto SP: Ploidy reduction in Saccharomyces cerevisiae. 2008, 4:91-94.

65. Gerstein AC, Otto SP: Ploidy and the Causes of Genomic Evolution. Journal of Heredity 2009, 100:571-581.

66. Gerstein AC, Otto SP: Cryptic Fitness Advantage: Diploids Invade Haploid Populations Despite Lacking Any Apparent Advantage as Measured by Standard Fitness Assays. PLOS ONE 2011, 6:e26599.

67. Gerstein AC, Cleathero LA, Mandegar MA, Otto SP: Haploids adapt faster than diploids across a range of environments. Journal of Evolutionary Biology 2011, 24:531-540.

68. Selmecki AM, Maruvka YE, Richmond PA, Guillet M, Shoresh N, Sorenson AL, De S, Kishony R, Michor F, Dowell R, et al.: Polyploidy can drive rapid adaptation in yeast. Nature 2015, 519:349-352.

69. Yona AH, Manor YS, Herbst RH, Romano GH, Mitchell A, Kupiec M, Pilpel Y, Dahan O: Chromosomal duplication is a transient evolutionary solution to stress. Proceedings of the National Academy of Sciences 2012, 109:21010-21015. *An example of the role of chromosomal duplications as initial evolutionary responses to stress, which are later followed by more "refined" adaptation. .

70. Dunham MJ, Badrane H, Ferea T, Adams J, Brown PO, Rosenzweig F, Botstein D: Characteristic genome rearrangements in experimental evolution of Saccharomyces cerevisiae. Proc Natl Acad Sci U S A 2002, 99:16144-16149.

71. Payen C, Di Rienzi SC, Ong GT, Pogachar JL, Sanchez JC, Sunshine AB, Raghuraman MK, Brewer BJ, Dunham MJ: The Dynamics of Diverse Segmental Amplifications in Populations of Saccharomyces cerevisiae Adapting to Strong Selection. G3: Genes|Genomes|Genetics 2014, 4:399-409. 
72. Sunshine AB, Payen C, Ong GT, Liachko I, Tan KM, Dunham MJ: The Fitness Consequences of Aneuploidy Are Driven by Condition-Dependent Gene Effects. PLoS Biol 2015, 13:e1002155. *Analysis of the role of aneuploidy in driving adaptation in experimentally evolved yeast populations. .

73. Gresham D, Desai MM, Tucker CM, Jenq HT, Pai DA, Ward A, DeSevo CG, Botstein D, Dunham MJ: The repertoire and dynamics of evolutionary adaptations to controlled nutrient-limited environments in yeast. PLoS Genet 2008, 4:e1000303.

74. Hong J, Gresham D: Molecular specificity, convergence and constraint shape adaptive evolution in nutrient-poor environments. PLoS Genet 2014, 10:e1004041.

75. Dunn B, Paulish T, Stanbery A, Piotrowski J, Koniges G, Kroll E, Louis EJ, Liti G, Sherlock G, Rosenzweig F: Recurrent Rearrangement during Adaptive Evolution in an Interspecific Yeast Hybrid Suggests a Model for Rapid Introgression. PLoS Genet 2013, 9:e1003366.

76. Lee M-C, Marx CJ: Repeated, Selection-Driven Genome Reduction of Accessory Genes in Experimental Populations. PLoS Genet 2012, 8:e1002651.

77. Lieberman TD, Flett KB, Yelin I, Martin TR, McAdam AJ, Priebe GP, Kishony R: Genetic variation of a bacterial pathogen within individuals with cystic fibrosis provides a record of selective pressures. Nat Genet 2014, 46:82-87.

78. Rainey PB, Travisano M: Adaptive radiation in a heterogeneous environment. Nature 1998, 394:69-72.

79. Rozen DE, Lenski RE: Long-Term Experimental Evolution in Escherichia coli. VIII. Dynamics of a Balanced Polymorphism. The American Naturalist 2000, 155:24-35.

80. Friesen ML, Saxer G, Travisano M, Doebeli M: Experimental evidence for sympatric ecological diversification due to frequency-dependent competition in Escherichia coli. Evolution 2004, 58:245-260.

81. MacLean RC: Adaptive radiation in microbial microcosms. J Evol Biol 2005, 18:1376-1386.

82. Helling RB, Vargas CN, Adams J: Evolution of Escherichia coli during Growth in a Constant Environment. Genetics 1987, 116:349-358.

83. Rozen DE, Philippe N, Arjan de Visser J, Lenski RE, Schneider D: Death and cannibalism in a seasonal environment facilitate bacterial coexistence. Ecol Lett 2009, 12:34-44.

84. Rosenzweig RF, Sharp R, Treves DS, Adams J: Microbial evolution in a simple unstructured environment: genetic differentiation in Escherichia coli. Genetics 1994, 137:903-917.

85. Le Gac M, Plucain J, Hindré T, Lenski RE, Schneider D: Ecological and evolutionary dynamics of coexisting lineages during a long-term experiment with Escherichia coli. Proceedings of the National Academy of Sciences 2012, 109:9487-9492.

86. Levy SF, Blundell JR, Venkataram S, Petrov DA, Fisher DS, Sherlock G: Quantitative evolutionary dynamics using high-resolution lineage tracking. Nature 2015, 519:181-186. **This important study introduces a novel lineage-tracking system which provides an alternative to whole-genome sequencing in analyzing the dynamics of adaptation. .

87. Farlow A, Long H, Arnoux S, Sung W, Doak TG, Nordborg M, Lynch M: The Spontaneous Mutation Rate in the Fission Yeast Schizosaccharomyces pombe. Genetics 2015.

88. Dillon MM, Sung W, Lynch M, Cooper VS: The Rate and Molecular Spectrum of Spontaneous Mutations in the GC-Rich Multichromosome Genome of Burkholderia cenocepacia. Genetics 2015, 200:935-946.

89. Jeon J, Choi J, Lee G-W, Dean RA, Lee Y-H: Experimental Evolution Reveals Genome-Wide Spectrum and Dynamics of Mutations in the Rice Blast Fungus, <italic>Magnaporthe oryzae</italic>. PLOS ONE 2013, 8:e65416.

90. Carroll SM, Marx CJ: Evolution after Introduction of a Novel Metabolic Pathway Consistently Leads to Restoration of Wild-Type Physiology. PLoS Genet 2013, 9:e1003427. 


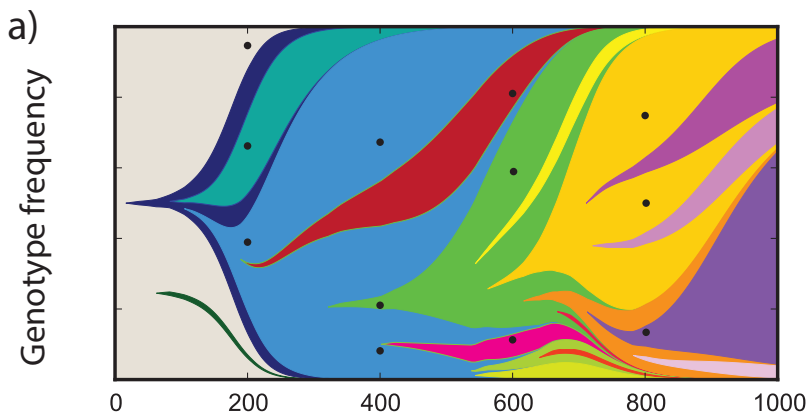

b) 1.0

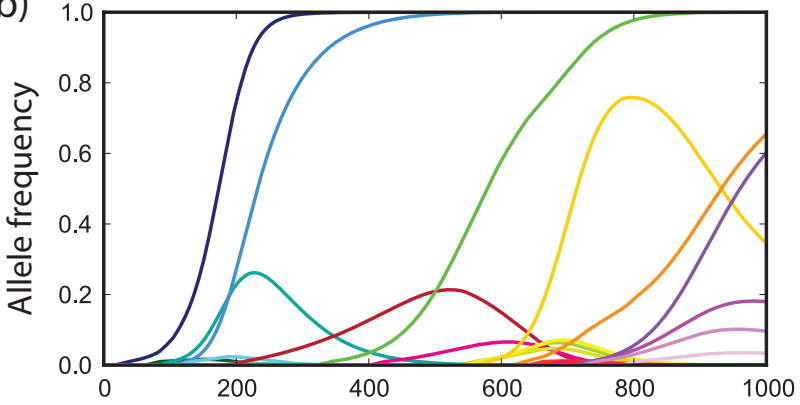

c)

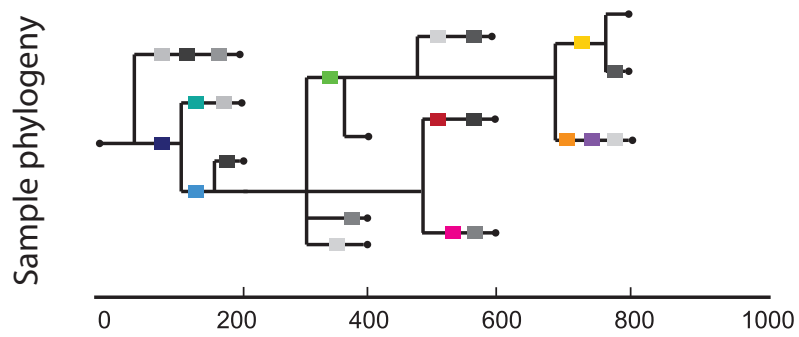

Generations 